\title{
Posterior reversible encephalopathy syndrome after combined spinal epidural anesthesia for intertrochanteric hip fracture repair
}

\section{Anna Spanomanoli, Triantafyllia Dimou, Stelios Michelis \\ Anesthesiology Department, Mediterraneo Hospital Athens, Greece}

Background: Posterior reversible encephalopathy syndrome (PRES) is a rare neurological disorder characterized by varied neurological symptoms, including headache, visual loss, mental abnormality, seizures and focal neurological deficits. The syndrome has been described as a post -procedural adverse effect in association with both general and spinal anesthesia.

Aim: To present the case of PRES in the PACU after hip fracture repair under spinal -epidural anesthesia.

\section{Methods/Results:}

An 85 -year-old hypertensive, ASA II male, was scheduled for intertrochanteric hip fracture surgery under combined spinal epidural anesthesia. Intravenous midazolam $2 \mathrm{mg}$ was given as premedication. In the operating room standard monitoring was established revealing a blood pressure (BP) of 225/125. After intrathecal injection of $10 \mathrm{mg}$ ropivacaine, BP successively dropped to $180 / 100$ and $165 / 95,90 / 65$ then remaining stable at $125 / 75$ throughout the remainder of the surgery.

After the 45 -minute operation, in the PACU, the patient presented with drowsiness, confusion, somnolence and diminished speech followed by vomiting and grand mal seizure 1 hour later. He was immediately transferred to High Dependency Unit (HDU) for further treatment and investigation. MRI scans showed increased fluid -attenuated inversion recovery (FLAIR) intensity in the cortical, parietal -occipital and temporal -occipital regions bilaterally, suggesting the diagnosis of PRES. The patient became responsive two days later and exited the HDU.

\section{Summary/ Conclusions:}

PRES should be considered in the differential diagnosis of patients with seizures in the postoperative period. Severe hypertension is a major predisposing factor. Most patients recover with full resolution of imaging findings and no neurological deficits.

\section{PREVALENCE OF CLINICAL SYMPTOMS AND SIGNS}

\begin{tabular}{|l|l|}
\hline SYMPTOMS & PREVALENCE \\
\hline Encephalopathy & $50-80 \%$ \\
\hline Seizure & $60-75 \%$ \\
\hline Headache & $50 \%$ \\
\hline Visual disturbances & $33 \%$ \\
\hline Focal neurological deficit & $10-15 \%$ \\
\hline Status epilepticus & $5-15 \%$ \\
\hline & S Stroke Cerebrovasc Dis 2012; 21: 873-82.
\end{tabular}

Stroke Cerebrovasc Dis 2012; 21 : 873-82.

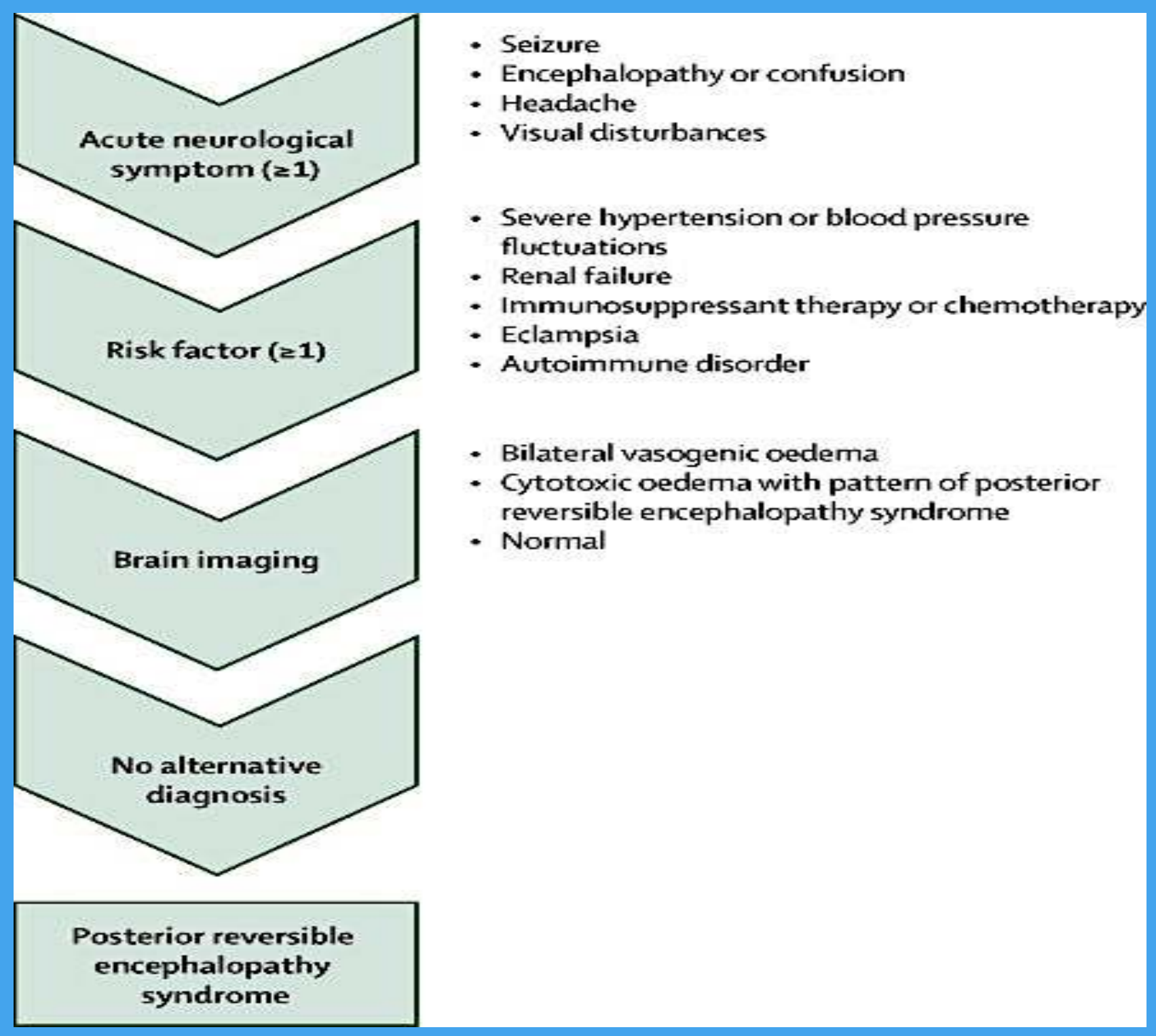

REGIONAL INVOLVEMENT BY PRES IN SERIES OF 136 PATIENTS

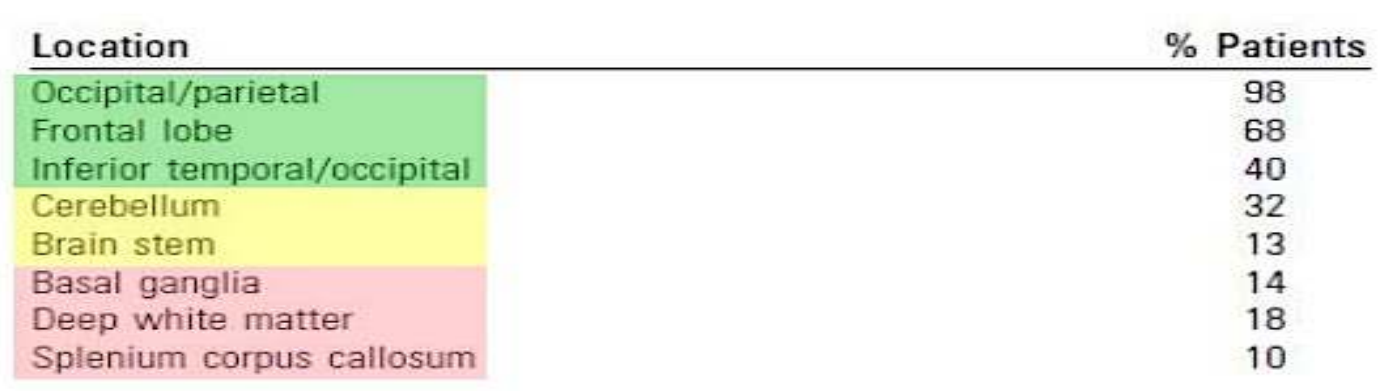

AJNR 2007;28:1302-07
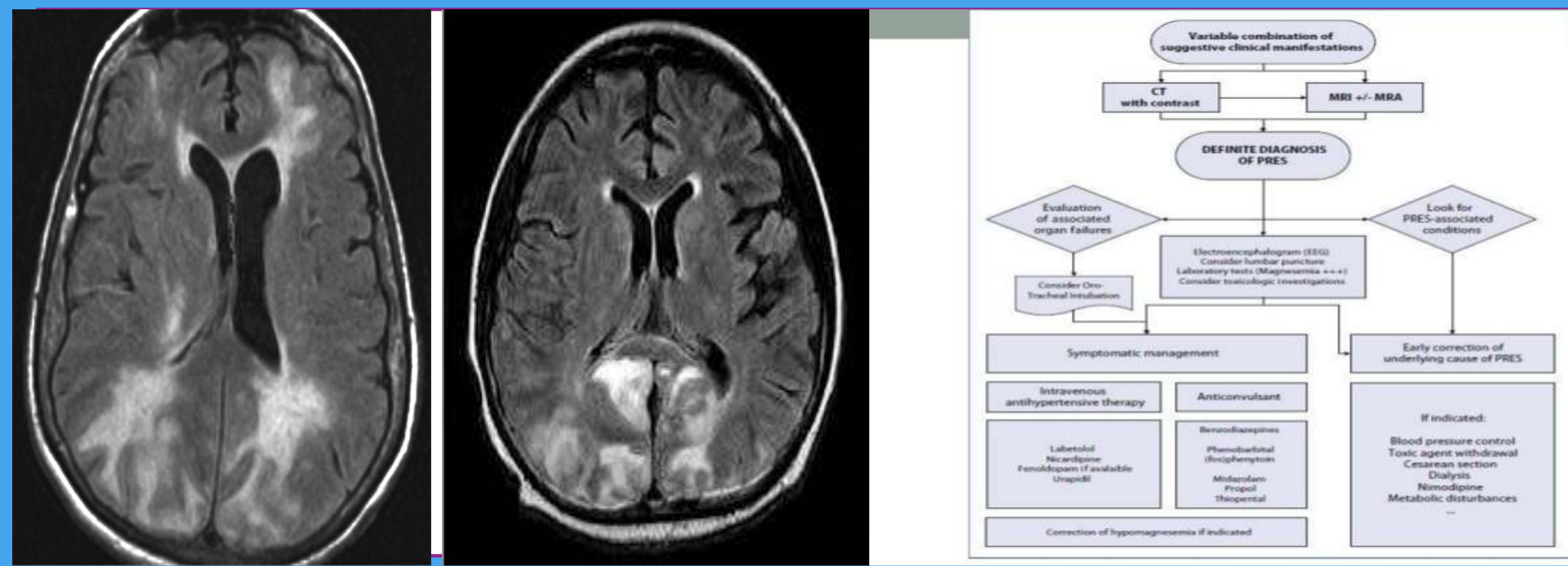